\title{
The intentionality bias and schizotypy
}

Authors: Moore, J. W.* ${ }_{a} \&$ Pope, A.a

Affiliations: a. Department of Psychology, Goldsmiths, University of London, SE14 6NW, UK

*Corresponding author:

Dr James Moore

Department of Psychology

Goldsmiths, University of London

Lewisham Way

London

SE14 6NW

Email: j.moore@gold.ac.uk

Tel: +44 (0)20 70785130

Running head: Intentionality bias and schizotypy 


\begin{abstract}
The 'intentionality bias' refers to our automatic tendency to judge other peoples' actions to be intentional. In this experiment we extended research on this effect in two key ways. First, we developed a novel non-linguistic task for assessing the intentionality bias. This task used video stimuli of ambiguous movements. Second, we investigated the relationship between the strength of this bias and schizotypy (schizophrenia-like symptoms in healthy individuals). Our results showed that the intentionality bias was replicated for the video stimuli and also that this bias is stronger in those individuals scoring higher on the schizotypy rating scales. Overall these findings lend further support for the existence of the intentionality bias. We also discuss the possible relevance of these findings for our understanding of certain symptoms of schizophrenic illness.
\end{abstract}

Key words: Intentionality bias; Intentions; Movement perception; Action observation; Schizotypy; Schizophrenia 


\section{Introduction}

The ability to distinguish between intentional and unintentional movements is integral to our social lives. Judging someone else's action to be intentional carries with it the assumption that they are also responsible. This clearly matters to us. Our response to finding someone reverse into our expensive new car will be different if we think it was because they did not see us, rather than because they did not like our ostentatious show of wealth. The importance of intentionality is also apparent in systems of law, where criminal responsibility rests on judgements of intentionality.

Our day-to-day assessments of intentionality seem to be effortless and there is strong agreement amongst adults concerning the kinds of movements that can be regarded as intentional (Malle \& Knobe, 1997). Yet despite the apparent ease with which we make these decisions, it has been suggested that there might be more to intention attribution than meets the eye. For example, Rosset (2008) has proposed a dual-process model of intention attribution in adults. On this view, intentional explanations of movement are automatically triggered and represent our default for explaining others' behaviour. Rosset called this the 'intentionality bias'. Furthermore, according to this dual-process model unintentional explanations for actions are only ever arrived at when intentional explanations are overridden by higher-level cognitive processes. These cognitive processes are thought to utilise knowledge of social norms, alternative causes and behavioural cues to arrive at alternative explanations for behaviour. Initial support for the dual-process view came from a series of experiments conducted by Rosset (2008). It was found that when participants read sentences describing an action, they tended to favour intentional interpretations, even when the action described was prototypically accidental. Moreover, this intentionality bias was augmented 
when participants were given less time to make their decision. It is thought that this occurred because cognitive resources were unable to be fully deployed in the speeded conditions.

In the present study we build upon this work in two key ways. First, we developed a novel paradigm for assessing the presence of the intentionality bias. The original work was based on written descriptions of actions. One significant limitation of these stimuli, as noted by Rosset, is that the intentionality bias may actually reflect a linguistic bias. That is, the wording of the sentences may encourage intentional attributions. In the present study we overcome this potential confound by using video stimuli. These were short video clips of movements that were ambiguous with respect to whether the movement was intentional or not. We investigated whether the intentionality bias would replicate with these novel stimuli. If it does then people should make more 'Intentional' judgements when viewing these ambiguous movement stimuli.

Second, we investigated the relationship between the intentionality bias and schizotypy. It has been suggested that psychotic experiences may lie on a continuum, with such experiences occurring in psychologically healthy individuals to a greater or lesser extent (Van Os, Linscott, Myin-Germeys, Delespaul, \& Krabbendam, 2009). Furthermore, previous studies have shown that cognitive performance in highly schizotypal individuals closely resembles performance of patients with a diagnosis of schizophrenia (e.g. Cochrane, Petch, \& Pickering, 2012). Given this apparent link between schizotypy and schizophrenia, the schizotypy dimension offers an invaluable research tool, allowing us to explore patterns of perception, experience and cognition redolent of schizophrenia, in a sample that is free of medication and hospitalisation, both of which can be deeply problematic confounds in patient samples (Moore, Dickinson, \& Fletcher, 2011). 
Our motivation for studying schizotypy in the context of the intentionality bias is that certain schizophrenic symptoms may be linked to deficits in intention attribution. For example, patients with paranoid delusions typically believe that an external force or agent is intentionally trying to harm them. It has been suggested that these delusions arise because patients have difficulty in both the understanding and the attribution of intentionality (Frith, 1992; 1994). This is supported by experimental work in which patients show a greater propensity to attribute intentionality to inanimate objects (e.g. Blakemore, Sarfati, Bazin, \& Decety, 2003). This suggests that patients with schizophrenia over-attribute intentions to others. If this were the case then they should show an exaggerated intentionality bias. We investigated this association in a non-clinical sample by measuring schizotypy.

\section{Methods}

\section{Participants}

35 participants took part in the experiment for course credit ( 24 females; mean age: 20 years; age range: 18-31). All had normal or corrected-to-normal vision.

\section{Stimuli}

\section{Movement stimuli}

There were 24 movement video stimuli in total. In each video clip participants saw, from front on, a hand with a finger strapped into a key (see Figure 1). After one of three delays (randomised across trials) the finger would move down on the key, depressing it fully, and return to the starting position. Participants were told beforehand that these movements could be either intentional or unintentional and that the videos across all trials would contain a mixture of both types of movement. Participants were told that if the movement was 
intentional, the agent actively pressed the button. If unintentional, the agent's finger was passively moved by a pulley device attached to the under side of the keyboard. In reality the same movement was presented on each trial and was, in fact, a passive unintentional movement (i.e. the actor's finger had been moved by the pulley during recording of the video). This perceptual equivalence across trials ensures that perceptual cues do not confound intention attribution judgements.

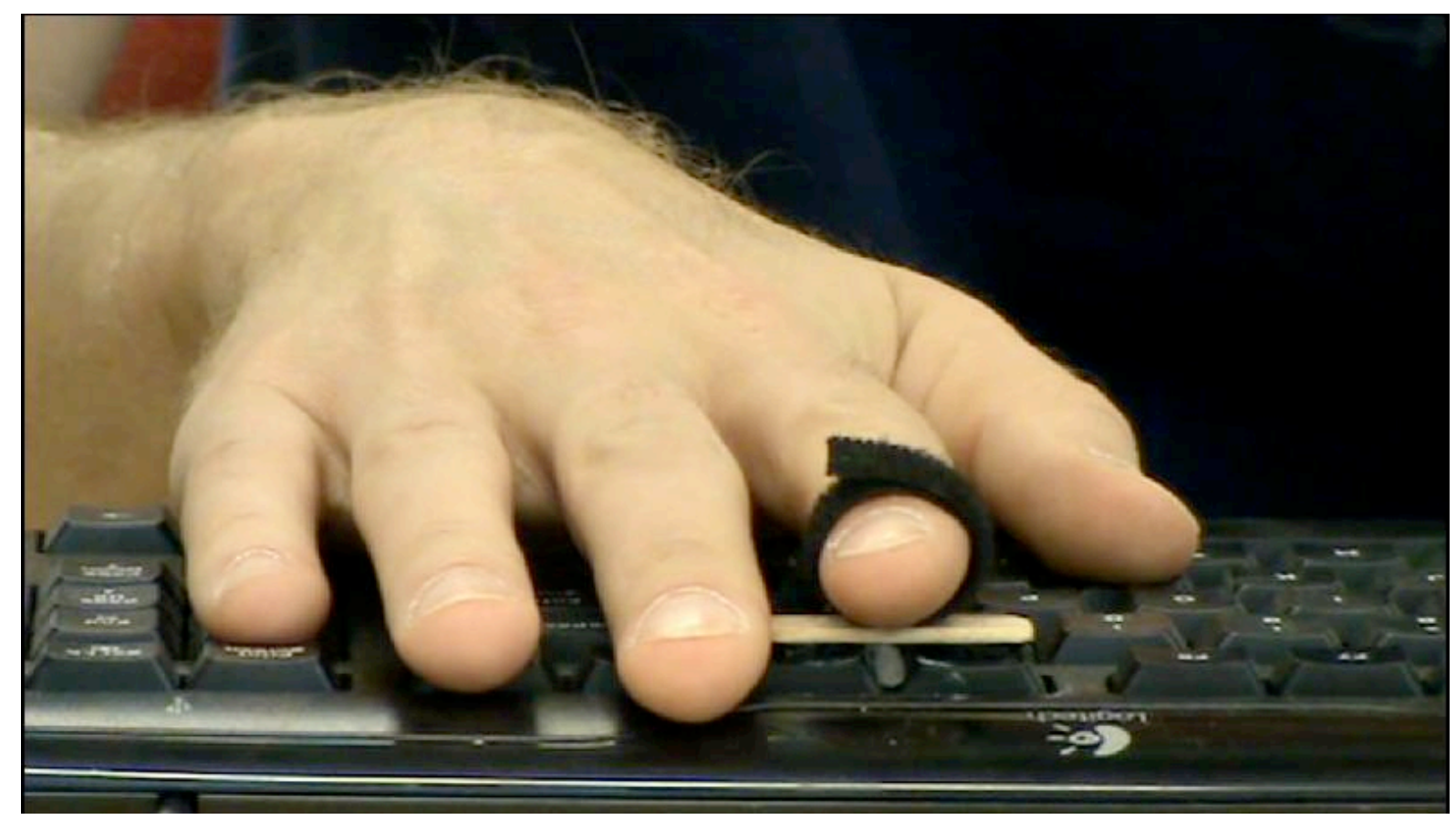

Figure 1. A still from the video stimulus used in this experiment.

\section{Schizotypy scales}

The Peters Delusion Inventory (PDI: Peters, Joseph, Day, \& Garety, 2004) is a 21-item scale designed to measure delusional ideation in the normal population (e.g. "Do your thoughts ever feel alien to you in some way?"). When an item is endorsed, three five-point scales exploring distress, preoccupation, and conviction are then completed.

The Cardiff Anomalous Perceptions Scale (CAPS: Bell, Halligan, \& Ellis, 2006) is a 32-item scale designed to measure unusual perceptual experiences in the normal population (e.g. "Do 
you ever see things that other people cannot?"). When an item is endorsed, three five-point scales exploring distress, intrusiveness, and frequency are then completed.

\section{Procedure}

Participants were seated in a computer cubicle testing room. Instructions and stimuli were presented on the computer in front of them. Participants were tested individually in the presence of an experimenter. All participants completed the intention attribution task first. The instructions told the participant that they would watch 26 short clips (two practice trials and 24 experimental trials) of an actor and simple key press movements on a keyboard. Their task on each trial was to report whether they thought that the movement they had just seen was intentional or unintentional. Each trial consisted of the presentation of a fixation cross (2 seconds) followed by the movie stimulus ( 2 seconds) followed by a judgement probe (4 seconds). Participants made their judgements verbally, and these were recorded by the experimenter.

Following the intention attribution task participants completed the two schizotypy questionnaires. The order of their completion was counterbalanced across participants (18 participants completed the PDI first).

\section{Results}

To calculate the intentionality bias score for each participant, the total number of 'Intentional' judgements was divided by the total number of trials (24) and multiplied by 100 to give a percentage score. To calculate the PDI and CAPS scores for each participant a 1 was scored for each 'Yes' response and this was summed across all items. Added to this were the scores for the participant's answers to the three additional 5-point scales for each 'Yes' response. 
Summary statistics are presented in Table 1. For inferential statistics, 2-tailed tests were used throughout.

Table 1. Summary statistics for the intentionality bias task, PDI and CAPS

\section{Mean (SD)}

Intentionality bias (\% 'Intentional') $64.2(17.6)$

PDI

$52.4(40.4)$

CAPS

$67.0(55.3)$

\section{Intentionality bias}

The mean intentionality bias score was $64.2 \%$ (SD: 17.6). A one-sampled t-test (with a test value of 50) on the intentionality bias scores shows that participants were significantly more likely to report that the movement they had just seen was intentional, $\mathrm{t}(34)=4.76, \mathrm{p}<.001$ (2-tailed). This is in line with the intentionality bias reported by Rosset (2008), suggesting that for ambiguous movement stimuli people are more likely to judge them to be intentional.

\section{Intentionality bias and schizotypy}

We were also interested in the relationship between individual differences in the intentionality bias and scores on the schizotypy scales. The scatterplots are shown in Figure 2. Two correlation analyses were performed (the significance level was Bonferroni-corrected to .025). Both total CAPS scores and total PDI scores were significantly correlated with the strength of the intentionality bias (CAPS: $r=.534, p=.001,2$-tailed; PDI: $r=.451, p=.006$, 2-tailed). This suggests that individual differences in the intentionality bias are related to the degree of schizophrenia-like symptoms in healthy adults. 
A)

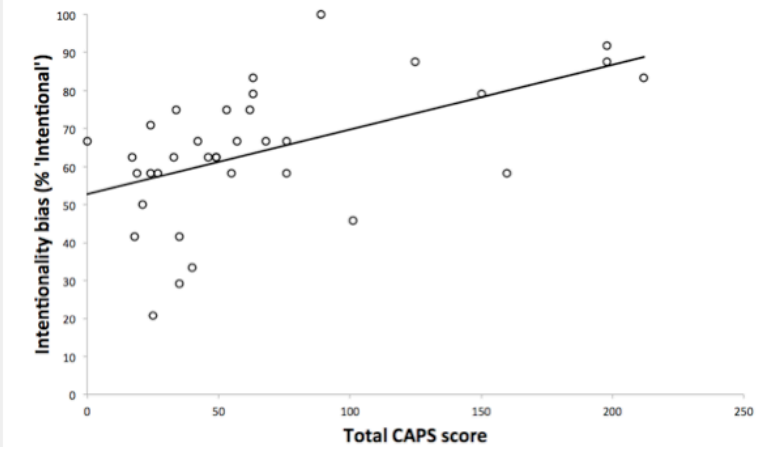

B)

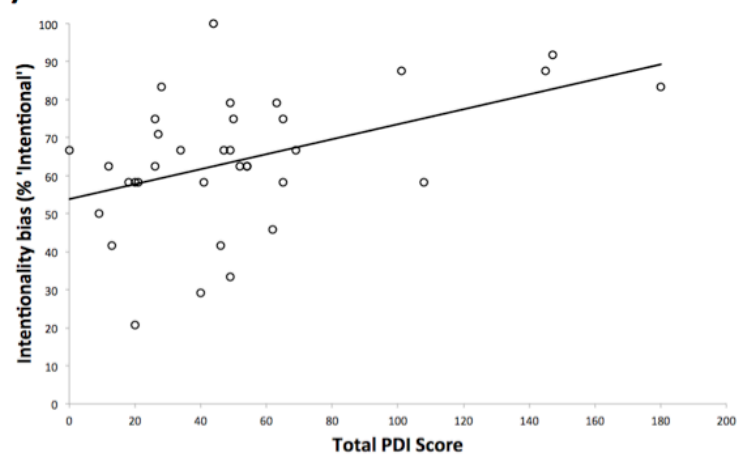

Figure 2. Scatterplots showing the relationship between intentionality bias and A) CAPS scores and B) PDI scores

\section{Intentionality bias and schizotypy sub-scores}

The schizotypy scales not only assess the occurrence of beliefs/ perceptual experiences but also include measures of degree. When an item is endorsed on the PDI, participants are also asked to indicate 'distress', 'preoccupation' and 'conviction'. Similarly when an item is endorsed on the CAPS, participants are asked to indicate 'distress', 'intrusiveness' and 'frequency'. It has been suggested that it is the degree of 'Distress' in particular that differentiates pathological from non-pathological beliefs and experiences (Corlett \& Fletcher, 2012; Peters et al., 2004; Sisti et al., 2012). In light of this, we also looked at the relation between the intentionality bias (the dependent variable) and total scores for each of the additional measures. We ran two separate hierarchical linear regression analyses for the PDI and CAPS respectively. Given the purported clinical significance of distress, for both analyses the 'Distress' sub-score was entered into the model first followed by the two remaining sub-scores (which were entered simultaneously into the linear regression model). The results are presented in Table 2. These results suggest that the 'Distress' score was a significant predictor of the intentionality bias. However, it is important to note that scores on the three sub-scales for both schizotypy measures were highly correlated with each other $(\mathrm{r}>$ 9), suggesting that 'Distress' itself might not uniquely predict the intentionality bias. 
Table 2. Output for separate hierarchical linear regression analyses assessing the relationship between PDI and CAPS sub-scores and the intentionality bias (the dependent variable)

\section{Beta Standard error of beta Standardised beta}

\section{PDI}

Step 1

\begin{tabular}{lll}
\hline Constant & 55.62 & 4.09
\end{tabular}

'Distress'

0.59

0.21

$.44^{*}$

Step 2

\begin{tabular}{lll}
\hline Constant & 54.24 & 4.69
\end{tabular}

$\begin{array}{llll}\text { 'Distress' } & 0.02 & 1.14 & .02\end{array}$

$\begin{array}{llll}\text { 'Preoccupation' } & 0.34 & 1.44 & .24\end{array}$

$\begin{array}{llll}\text { 'Conviction' } & 0.28 & 0.73 & .20\end{array}$

\section{CAPS}

\begin{tabular}{llll}
\hline Step 1 & & & \\
\hline Constant & 54.18 & 3.64 & $.55^{*}$ \\
'Distress' & 0.53 & 0.14 & 1.54 \\
\hline Step 2 & & & \\
& & & \\
Constant & 55.26 & 4.12 & -1.25 \\
'Distress' & 1.47 & 0.76 & .25 \\
'Intrusiveness' & -1.19 & 0.71 & \\
'Frequency' & 0.29 & 0.57 & \\
\hline $\begin{array}{l}\text { Note: PDI: }{ }^{2}=.20 \text { for Step } 1, \Delta \mathrm{R}^{2}= \\
.06 \text { for Step 2. }\end{array}$ & $* 0.01$ for Step 2 / CAPS: PDI: $\mathrm{R}^{2}=.31$ for Step $1, \Delta \mathrm{R}^{2}=$
\end{tabular}




\section{Discussion}

The intentionality bias refers to the tendency for people to interpret the behaviour of others as intentional (Rosset, 2008). In the present study we found that the intentionality bias holds for videos of ambiguous movements and that the magnitude of the intentionality bias is related to individual differences in schizotypy.

The first aim of this study was to investigate the presence of this bias for non-linguistic stimuli. In the original study by Rosset (2008) participants read descriptions of actions and made intentionality judgements concerning them. As noted by Rosset, it is possible that the observed intentionality bias represents a linguistic bias. This is something we addressed in the present study, replicating the intentionality bias for video stimuli depicting ambiguous movements. This finding suggests that the previously reported intentionality bias is not simply an artefact of the linguistic stimuli used.

This result lends further support to the dual-process view of intention attribution. That is, our findings support the idea that when observing action our default is to interpret the behaviour as intentional. It is not clear why this default is present. Bègue, Bushman, Giancola, Subra and Rosset (2010) have suggested that judging an action to be intentional is computationally less demanding. This is because judging an action to be unintentional requires one to search for other possible causes. This would fit with Rosset's (2008) finding that reducing the involvement of higher-level cognitive processes further accentuates the intentionality bias. Although speculative, we would also suggest that the intentionality bias could confer an advantage by virtue of the fact that it may be better to make a false positive error (believing something was caused intentionally by an agent when in fact it was an accident), than a false negative error (believing something was accidental when in fact it was intentionally caused). 
In doing so we are primed to deal with the putative involvement of another agent who may or may not pose a threat. Indeed it is perhaps informative that the intentionality bias might be most pronounced for negative events (Knobe, 2003). Our suggestion is consistent with the Error Management Theory of cognitive biases (Haselton \& Buss, 2000; Haselton \& Nettle, 2006). The assumption here is that cognitive biases arise under conditions of uncertainty where the costs false positive and false negative errors are not equivalent. In the context of the intentionality bias, we propose that the cost of a false positive is less than the cost of a false negative.

Further to offering support for the intentionality bias, our novel paradigm may have the additional benefit of providing a way of testing the intentionality bias in individuals for whom linguistic stimuli may be problematic. For example, it may not be possible to use linguistic stimuli in younger children or those with language impairments. In this way, our paradigm (and further modifications of it) may help widen the scope of research on the intentionality bias.

The second aim of this study was to investigate the relationship between the intentionality bias and schizotypy. This was motivated by apparent deficits in intention attribution associated with schizophrenic illness (e.g. Blakemore et al., 2003). We found that individuals scoring higher on the schizotypy scales showed a stronger intentionality bias. Before interpreting this finding it is interesting to note that individuals scoring higher on schizotypy scales also show a more liberal 'acceptance bias' (Corlett et al., 2009). That is, they have a tendency to make false alarm errors. Therefore, one possibility is that this liberal acceptance bias could explain the relationship between schizotypy and the intentionality bias - perhaps the tendency towards false positives makes people more likely to perceive intentionality 
where there is none. However, this explanation is unlikely given our experimental design. Participants in our experiment had a choice between two alternatives - intentional vs. unintentional movement - and were also explicitly told that the trials would contain a mixture of the two types of movement. In this way, we would suggest that a liberal acceptance bias is unable to explain why participants favoured one judgement over the other on this task.

These results therefore do suggest that an exaggerated intentionality bias may be a relevant information-processing deficit in certain sub-types of schizophrenic illness. For example, the persecutory delusions held by some patients may be explicable in terms of an exaggerated intentionality bias, with these patients being more prone to seeing events that happen to them as intentionally caused. However, more work is needed to ascertain what specific aspects of schizotypy/ schizophrenia (if any) this exaggerated intentionality bias is related to. Future work should therefore consider other aspects not measured here, such as bodily changes, anhedonia and disorganisation.

Finally, the observed relationship between schizotypy and the intentionality bias is also intriguing given the so-called 'hypo-frontality' exhibited by patients with schizophrenia. This reduced frontal activity is thought to be responsible for deficits in cognitive control and executive function found in patients (Weinberger, 1988). There is also some evidence of frontal deficits in highly schizotypal individuals (although this is more equivocal). For example, behavioural studies of executive function show poorer performance in these individuals (Park, Holzman, \& Lenzenweger, 1995; Park \& McTigue, 1997) and some neuroimaging studies have indicated frontal abnormalities (e.g. Raine, Sheard, Reynolds, \& Lencz, 1992). The exaggerated intentionality bias we find in highly schizotypal individuals is therefore perhaps not surprising given that these higher-level cognitive processes are thought 
to be necessary for unintentional explanations of behaviour (Rosset, 2008). If these processes are disrupted then the intentionality bias should be more pronounced.

\section{Conclusions}

Overall, our results support the notion of an intentionality bias underlying our perception of other peoples' movements. We extend previous work by firstly developing a novel paradigm. This opens up possibility of testing for this bias in different groups. Furthermore, the finding that this bias is linked to individual differences in schizotypy provides a tentative insight into a possible information processing abnormality that might help explain certain symptoms of schizophrenic illness. 


\section{References}

Bègue, L., Bushman, B. J., Giancola, P. R., Subra, B., \& Rosset, E. (2010). “There Is No Such Thing as an Accident," Especially When People Are Drunk. Personality and Social Psychology Bulletin, 36(10), 1301-1304.

Bell, V., Halligan, P. W., \& Ellis, H. D. (2006). The Cardiff Anomalous Perceptions Scale (CAPS): A new validated measure of anomalous perceptual experience. Schizophrenia Bulletin, 32(2), 366-377.

Blakemore, S.-J., Sarfati, Y., Bazin, N., \& Decety, J. (2003). The detection of intentional contingencies in simple animations in patients with delusions of persecution. Psychological Medicine, 33(8), 1433-1441.

Cochrane, M., Petch, I., \& Pickering, A. D. (2012). Aspects of cognitive functioning in schizotypy and schizophrenia: evidence for a continuum model. Psychiatry Research, 196(2), 230-234.

Corlett, P R, \& Fletcher, P. C. (2012). The neurobiology of schizotypy: fronto-striatal prediction error signal correlates with delusion-like beliefs in healthy people. Neuropsychologia, 50(14), 3612-3620. doi:10.1016/j.neuropsychologia.2012.09.045

Corlett, P. R., Simons, J. S., Pigott, J., Gardner, J. M., Murray, G. K., Krystal, J. H., \& Fletcher, P. C. (2009). Illusions and delusions: relating experimentally-induced false memories to anomalous experiences and ideas. Frontiers in Behavioral Neuroscience, 3,53 .

Frith, C. (1994). Theory of mind in schizophrenia. Retrieved from http://psycnet.apa.org/psycinfo/1994-97485-008

Frith, C. D. (1992). The cognitive neuropsychology of schizophrenia. Psychology Press. 
Haselton, M. G., \& Buss, D. M. (2000). Error management theory: a new perspective on biases in cross-sex mind reading. Journal of Personality and Social Psychology, $78(1), 81$.

Haselton, M. G., \& Nettle, D. (2006). The paranoid optimist: An integrative evolutionary model of cognitive biases. Personality and Social Psychology Review, 10(1), 47-66.

Knobe, J. (2003). Intentional action in folk psychology: An experimental investigation. Philosophical Psychology, 16(2), 309-324.

Malle, B. F., \& Knobe, J. (1997). The folk concept of intentionality. Journal of Experimental Social Psychology, 33(2), 101-121.

Moore, J. W., Dickinson, A., \& Fletcher, P. C. (2011). Sense of agency, associative learning, and schizotypy. Consciousness and Cognition, 20(3), 792-800.

Park, S., Holzman, P. S., \& Lenzenweger, M. F. (1995). Individual differences in spatial working memory in relation to schizotypy. Journal of Abnormal Psychology, 104(2), 355.

Park, S., \& McTigue, K. (1997). Working memory and the syndromes of schizotypal personality. Schizophrenia Research, 26(2-3), 213-220. doi:10.1016/S09209964(97)00051-0

Peters, E., Joseph, S., Day, S., \& Garety, P. (2004). Measuring Delusional Ideation. Schizophrenia Bulletin, 30(4), 1005-1022.

Raine, A., Sheard, C., Reynolds, G. P., \& Lencz, T. (1992). Pre-frontal structural and functional deficits associated with individual differences in schizotypal personality. Schizophrenia Research, 7(3), 237-247. doi:10.1016/0920-9964(92)90018-Z

Rosset, E. (2008). It's no accident: Our bias for intentional explanations. Cognition, 108(3), 771-780. 
Sisti, D., Rocchi, M. B. L., Siddi, S., Mura, T., Manca, S., Preti, A., \& Petretto, D. R. (2012). Preoccupation and distress are relevant dimensions in delusional beliefs.

Comprehensive Psychiatry, 53(7), 1039-1043. doi:10.1016/j.comppsych.2012.02.005

Van Os, J., Linscott, R. J., Myin-Germeys, I., Delespaul, P., \& Krabbendam, L. (2009). A systematic review and meta-analysis of the psychosis continuum: evidence for a psychosis proneness-persistence-impairment model of psychotic disorder. Psychological Medicine, 39(2), 179.

Weinberger, D. R. (1988). Schizophrenia and the frontal lobe. Trends in Neurosciences, 11(8), 367-370. doi:10.1016/0166-2236(88)90060-4 